\title{
Severe Phenotype of De Barsy Syndrome in Two Siblings with Novel Mutations in the ALDH18A1 Gene
}

\author{
Smigiel $\mathbf{R}^{1 *}$, Kusmierska $\mathrm{K}^{2}$, Pollak $\mathrm{A}^{3}$, Szmyd $\mathrm{K}^{4}$, Kosinska $\mathrm{J}^{5}$, Polawski $\mathrm{T}^{2}$, Kostrzewa $\mathbf{G}^{6}$, Sasiadek $\mathbf{M M}^{7}$ and Ploski $\mathbf{R}^{5 *}$ \\ ${ }^{1}$ Department of Paediatrics and Rare Disorders, Wroclaw Medical University, Wroclaw, Poland \\ ${ }^{2}$ Department of Screening Study, Institute of Mother and Child, Warsaw \\ ${ }^{3}$ Department of Genetics, Institute of Physiology and Pathology of Hearing, Warsaw, Poland \\ ${ }^{4}$ Lower Silesia Children's Hospice, Wroclaw, Poland \\ ${ }^{5}$ Department of Medical Genetics, Warsaw Medical University, Warsaw, Poland \\ ${ }^{6}$ Department of Forensic Medicine, Warsaw Medical University, Warsaw, Poland \\ ${ }^{7}$ Department of Genetics, Wroclaw Medical University, Wroclaw, Poland
}

\begin{abstract}
De Barsy syndrome is a rare autosomal recessive genetic disorder characterized by growth retardation, intellectual disability, a prematurely-aged appearance (progeroid features) and loose skin (cutis laxa) as well as eye abnormalities and others. Some cases of de Barsy syndrome have been linked with mutations PYCR 1 or ALDH18A1. We describe a family with two siblings with clinically severe de Barsy syndrome in whom two novel mutations in ALDH18A1 (p.Glu100* and p.Arg724His) were found by clinical exome sequencing using TruSight One panel. The p.Glu100* is a novel mutation predicted to cause absence of the protein. The p.Arg724His has been found with low frequency $(0.000016)$ but not in association with human disease; it has been scored as pathogenic by CADD, MetaSVM, Polyphen2, MutationAssessor, SIFT and MutationTaster. The level of ammonia in serum was determined in second sibling and was in normal range. Amino acid profile in serum revealed decreased concentration of arginine, cytruline, homocysteine, PHE and ornithine. The patients suffer from severe symptoms of GERD such as vomiting, feeding problems instead of multistage therapy including Nissen fundoplication procedure as well as from epilepsy requires complex multidrug therapy. L-Arginine $(200 \mathrm{mg} / \mathrm{kg})$ and citrulline $(100 \mathrm{mg} / \mathrm{kg})$ were supplemented in the second sibling. The disease leads to premature apoptosis, so antioxidants (coenzyme Q, vitamin A and E) as well as carnitine were supplemented but without spectacular clinical results.
\end{abstract}

We provide clinical description of severe phenotype of de Barsy syndrome. Our molecular report broadens the spectrum of $A L D H 18 A 1$ mutations causing de Barsy syndrome.

Keywords: De Barsy syndrome; ALDH18A1 gene; Recessive mutations; Amino acids profile; Severe developmental delay

\section{Introduction}

De Barsy syndrome known as De Barsy-Moens-Dierckx syndrome is an ultrarare, autosomal recessive disease characterized by distinctive, dysmorphic facial features suggesting in the neonatal period progerialike appearance, cutis laxa, ocular defects and orthopedic abnormalities as well as athetoid movements, developmental delay and intellectual disability [1-4]. Whereas the genetic defect in de Barsy syndrome cannot be always established, the known causes are recessive mutations in PYCR1 gene (OMIM179035), encoding pyrroline-5-carboxylate reductase 1 as well as ALDH18A1 gene (OMIM138250), encoding D1-pyrroline-5carboxylate synthase (P5CS) [5-7]. Defects of the ALDH18A1 and PYCR1 genes also cause separate entities such as autosomal recessive cutis laxa type I (OMIM219100), autosomal recessive cutis laxa type II (ARCL2; OMIM219200), wrinkly skin syndrome (WSS; OMIM278250), gerodermia osteodysplastica (OMIM231070) or hyperammonemia, hypoornithinemia, hypocitrullinemia, hypoargininemia and hypoprolinemia [8,9].

We have ascertained a family with two siblings with clinical diagnosis of severe de Barsy syndrome molecularly confirmed by identification of two novel mutations in the ALDH18A1 gene.

\section{Clinical Report}

First proband was the third male child ofhealthynon-consanguineous couple and was born at 38 weeks of gestation by natural delivery after complicated pregnancy (hypotrophy) with following birth parameters: weight $2460 \mathrm{~g}(-2,1 \mathrm{SD})$, length $50 \mathrm{~cm}$, OFC $31 \mathrm{~cm}$, Apgar score 9 points. After birth hypotonia, feeding and sucking problems as well as facial dysmorphism suggesting progeria-like appearance, irregular lack of subcutaneous tissue, prominent vessels on the skin and wrinkling skin were observed. Moreover, severe GERD was diagnosed requiring Nissen fundoplication procedure and gastrostomy. Developmental milestones were severely delayed. The neurological examination showed spasticity and severe athetoid movements involving limbs and head. With time the abnormal movements were exaggerated together with hypersensitivity to the touch and anxiety.

Brain NMR study showed hypoplasia of corpus callosum. Ophthalmological examination revealed cloudy corneas; during re-consultation bilateral cataract were diagnosed needed surgical procedure. Hearing test showed bilateral deafness (70dB). EEG results performed several times were abnormal, but revealed no specific pattern that could suggests a particular the type of epilepsy.

*Corresponding author: Robert Smigiel, Department of Pediatrics, Wroclaw Medical University, PL 50-368 Wroclaw, Bartla 5, Poland, Tel: 00487178412 56; Fax: 00487178400 63; E-mail: robert.smigiel@umed.wroc.pl

Rafał Ploski, Department of Medical Genetics, Warsaw Medical University, PL 02103 Warsaw, Pawinskiego 3c, Poland, Tel: 00482257206 95; Fax: 00482257206 96; E-mail: rploski@wp.pl

Received December 24, 2016; Accepted January 24, 2017; Published January 31, 2017

Citation: Smigiel R, Kusmierska K, Pollak A, Szmyd K, Kosinska J, et al. (2017) Severe Phenotype of De Barsy Syndrome in Two Siblings with Novel Mutations in the ALDH18A1 Gene. J Clin Med Genomics 5: 145. doi: 10.4172/2472-128X.1000145

Copyright: $\odot 2017$ Smigiel R, et al. This is an open-access article distributed under the terms of the Creative Commons Attribution License, which permits unrestricted use, distribution, and reproduction in any medium, provided the original author and source are credited. 
Facial dysmorphism was present including microcephaly, sharpened facial features, large fontanelles, shallow eyeballs and prominent eyes, wrinkling skin in periorbital region, infra-orbital creases, small, short nose, long philtrum and prominent ears. Moreover, atrophy of subcutaneous tissue, dry and thin skin, wrinkling skin, especially on feet and hands as well as diffuse and protuberant abdomen. The child died at the age of 2 years with respiratory and circulatory insufficiency.

The second proband was a male born to the same parents at 38 weeks with hypotrophy (weigh birth $2040 \mathrm{~g},-2,3 \mathrm{SD}$ ). The same clinical symptoms were observed as in the older brother: hypotrophy, hypotony, sucking and feeding problems, facial and skin dysmorhism. The boy was operated on because of bilateral inguinal hernias and GERD (Nissen fundoplication and gastrostomy procedures were performed). Severe feeding problems, failure of thrive, permanent vomiting, psychomotor and developmental delay with hypotony, facial and skin dysmorphism, severe athetoid movements and lack of social contact were observed. Additional examinations revealed: abnormal EEG (variably localized changes alternately), vision problem in VEP study, hypoplasia of corpus callosum in MRI of brain, cloudy corneas (bilateral cataract) in ophthalmological examinations. The child requires complex multidrug therapy. Treatment with valproic acid and levetiracetam led to no clinical improvement. Replacement of valproic acid with lamotrigine improved neither the seizures (reduction in seizure frequency) nor the EEG (electroencephalography) pattern reaching a plateau in terms of severity of the clinical condition and then remaining stable.

Then clinical suspicion of de Barsy syndrome was considered.

\section{Genetic Studies}

Written informed consent was obtained prior to genetic testing from all subjects or their legal guardians.

The chromosomal analysis of first proband revealed a normal male karyotype (46,XY). PLP1 mutation screening (MLPA: Multiplex Ligation-Dependent Probe Amplification, direct sequencing) didn't revealed any abnormality. Moreover, no subtelomeric aberrations or interstitial microdeletion syndromes were found on MLPA testing (P070-A2 human telomere-5, P245-A2 microdeletion syndromes-1). Next, array-comparative genomic hybridization (array-CGH) was performed and showed two microduplications (Agilent $180 \mathrm{~K}$, hg 18): $5 \mathrm{q} 23.3$ (0,9 kbp) including the FBN2 gene and $13 \mathrm{q} 13.23(0,5 \mathrm{kbp})$ including NBEA gene. The identified microduplications were not considered as a pathogenic according to literature; however parents' studies did not disclose any of these alterations. Since this could suggest de novo events array CGH study was performed in the second proband but it did not disclose any abnormalities.

NGS analysis was performed using TruSight One kit according to manufacturer instructions (Illumina). The sample was run on $1 / 4$ of lane on HiSeq 1500 using 2x100 bp paired-end reads. Bioinformatics analysis was performed as previously described [10]. Briefly, after initial processing with CASAVA, the sequencing reads were aligned to the hg19 reference genome with Burrows-Wheeler Alignment Tool and further processed by Genome Analysis Toolkit [11]. Base quality score recalibration, indel realignment, duplicate removal and the SNP/ INDEL calling were done as described [12]. The detected variants were annotated using Annovar and converted to MS Access format for final manual analyses. Alignments were viewed with Integrative Genomics Viewer $[13,14]$. The min. 20x and 10x coverage of the target was $98.4 \%$ and $99.5 \%$, respectively.

Sanger sequencing was performed using BigDye Terminators kit v 3.1 (Life Technologies) with the following primers: forward 5' GGC ATG CAT TTC TGC ATA GTT 3', Reverse: 5' GCA ATT GCT GCT CTT GAG TG 3').

NGS analysis generated 97,099,776 reads. We filtered the results to retain high quality variants changing protein coding sequence or affecting splice sites with population frequency $<0.01$ (according to the EXAC database and an in house database of $\sim 500$ Polish exomes). After filtering there were 247 variants left. These variants were searched for biallelic mutations consistent with autosomal recessive inheritance as well as for hemizygous variants potentially causative of recessive sexlinked condition or heterozygous variants consistent with autosomal dominant de novo mutation (here we considered variants not found in available databases and predicted to cause loss of protein function, i.e., introduce premature stop codon or affect a splice site). The variants left after filtering are shown in Table 1.

Based on the known association between ALDH18A1 and de Barsy syndrome we strongly prioritized two heterozygous mutations in this gene: chr10:097402754-C>A, NM_001017423.1: p.Glu100*/c.298G>T and chr10:097369983-C>T, NM_001017423.1: p.Arg724His. The: p.Glu100* variant has not been reported before and the SNPeff software predicted that it leads to nonsense mediated decay (NMD) of mRNA and thus causes loss of function. The p.Arg724His variant (rs773714478) has not been previously associated with human disease has but it is present in ExAC database with frequency of 0.000016 . The p.Arg724His mutation has been scored as pathogenic by CADD (score $=34)$, MetaSVM (D), Polyphen2 (D), MutationAssessor (H), SIFT (D) and MutationTaster (D). The identified ALDH18A1 mutations were confirmed by standard Sanger sequencing. Both parents were tested and single mutation was observed in mother (p.Glu100*) as well as in father (p.Arg724His) indicating inherited origin of the variants. Testing of the affected brother revealed the same genotype as in the proband, i.e., presence of both mutations. Testing of healthy siblings was refused by the parents.

We also considered the p.Leu71* variant in PDK3 as possibly contributing to disease (Table 1) given that a mutation in this gene (p.R158H) has been described by Kennerson et al. (PubMed: 23297365) as causing X-linked dominant Charcot-Marie-Tooth disease-6 (CMTX6; OMIM: 300905). Sanger sequencing confirmed the hemizygous PDK3 p.Leu $71^{\star}$ variant in the proband and showed that it was inherited from the heterozygous mother. The affected brother of the proband did not have the PDK3 p.Leu71* variant. We provide evidence suggesting nonpathogenicity of loss of function variants in $P D K 3$.

\section{Metabolic and Biochemical Results}

Metabolic test such as GC/MS (gas chromatography-mass spectrometry) and tandem mass spectrometry (tandem MS), VLCFA level, CDG screening were normal in both patients. During the diagnostic process amino acids profile and ammonia level in serum were not assayed. Afterwards, when mutations in ALDH18A1 gene were identified, the level of ammonia in serum was determined in second sibling and was in normal range $(69,22 \mathrm{umol} / \mathrm{l}$ and 50,6 umol/l). Amino acid profile in serum revealed decreased concentration of arginine $(22,8$ umol/l, normal range: 46-128 umol/l), cytruline (11 umol/l, normal range: $16-46 \mathrm{umol} / \mathrm{l})$, homocysteine (1,2 umol/l, normal range: $3,3-8,3$ umol/l), PHE (33 umol/l, normal range: 39-74 umol/l) and ornitine (7,6 umol/l, normal range: $27-98 \mathrm{umol} / \mathrm{l})$. The remaining amino acids were in normal range including glicyne. Vitamin B12 level $(825 \mathrm{pg} / \mathrm{ml})$, folic acid level $(18 \mathrm{pg} / \mathrm{ml})$ were decreased. 


\begin{tabular}{|c|c|c|c|c|}
\hline Gene & Position & ID & Effect & Disease (inheritance, comments) \\
\hline \multicolumn{5}{|c|}{ Potentially AR (potentially biallelic variants, frequency $<0.01$ in available databases) } \\
\hline \multirow[t]{2}{*}{$A L D H 18 A 1$} & chr10:97402754 & - & NM_001017423.1:p.Glu100*/c.298G>T & $\begin{array}{l}\text { De Barsy syndrome or autosomal recessive cutis laxa } \\
\text { type }\end{array}$ \\
\hline & chr10:97369983 & rs773714478 & $\begin{array}{l}\text { NM_001017423.1:p.Arg724His/c.2171G }>\text { A, scored as } \\
\text { pathogenic by CADD (score=34), MetaSVM (D), Polyphen2 (D), } \\
\text { Mutation Assessor (H), SIFT (D) and MutationTaster (D) }\end{array}$ & III (http://omim.org/entry/219150) \\
\hline NLRC5 & chr16:57059318 & rs148647729 & $\begin{array}{l}\text { NM_032206.4:p.Ala155Thr/c.463G>A, apparently homozygous } \\
\text { ( } 5 \text { reads), scored as benign by CADD, MetaSVM, Polyphen2, } \\
\text { MutationAssessor, SIFT and MutationTaster }\end{array}$ & $\begin{array}{l}\text { Hematopoietic and immune system's phenotypes in } \\
\text { homozygous knock-out mice (http://www.informatics. } \\
\text { jax.org) }\end{array}$ \\
\hline \multirow[t]{2}{*}{ FAM186A } & chr12:50747016 & rs200241170 & NM_001145475.1:p.Ala1200Asp/c.3599C>A & $\begin{array}{l}\text { High }(>100) \text { frequency of loss of function alleles and } \\
\text { homozygotes in ExAC database }\end{array}$ \\
\hline & chr12:50744108 & rs113065589 & NM_001145475.1:c.6504G>C,NM_001145475.1:c.6503+4G>C & (http://exac.broadinstitute.org/gene/ENSG00000185958) \\
\hline \multicolumn{5}{|c|}{ Potentially AD (freq. 0 in available databases, predicted loss of function) } \\
\hline ATP5G1 & chr17:46970814 & - & NM_001002027.1:p.Ala12_Leu13fs/c.36_37insTCTG & None $(\mathrm{pLI}=0.33)$ \\
\hline CRLS1 & chr20:6012011 & - & NM_001127458.1:p.Thr120fs/c.359delC & None $(\mathrm{pLI}=0.03)$ \\
\hline KIF12 & chr9:116854866 & - & NM_138424.1:p.Trp413*/c.1239G>A & None (pLI=1.78E-05) \\
\hline \multicolumn{5}{|c|}{ Potentially $X$ liked (freq. 0 in available databases, predicted loss of function) } \\
\hline PDK3 & chrX:24512964 & - & NM_001142386.2:p.Leu71*/c.212T>A & Charcot-Marie-Tooth disease (X-linked dominant) \\
\hline
\end{tabular}

pLI: Probability that a gene is intolerant of loss-of-function variation (http://exac.broadinstitute.org)

Table 1: Variants found by NGS in the proband that were considered as potentially disease-causing.

\section{Discussion}

We have ascertained a family with two male siblings with clinical diagnosis of de Barsy syndrome molecularly confirmed by identification of two novel mutations in the ALDH18A1 gene: p.Glu100* and p.Arg724His (rs773714478) in both brothers. The p.Glu100* variant has not been reported before and it is predicted to cause absence of the protein. The p.Arg724His has been found with very low frequency (0.000016) but not in association with human disease. The p.Arg724His variant is likely to significantly decrease the protein function as it has been scored as pathogenic by CADD, MetaSVM, Polyphen2, MutationAssessor, SIFT and MutationTaster (D).

Both patients reported here had very similar disease presentation including birth hypotonia, feeding and sucking problems, progeria-like appearance, spasticity with severe athetoid movements and anxiety, hypoplasia of corpus callosum, cataracts needed surgical procedure, bilateral hearing impairment and abnormal ECG with severe seizures [1-9]. Biochemical studies performed in serum in the second affected sib showed normal level of ammonia whereas concentration of arginine, cytruline, homocysteine, PHE and ornitine were decreased. These symptoms are typical of de Barsy syndrome however; the disease in our patients was relatively severe leading to early death of older sibling. It is likely that the severity of the disease is related to the strong damaging effect of the observed $A L D H 18 A 1$ mutations $[7-9,15,16]$. In the second sibling seizures were treated by two epileptic drugs: valproic acid, phenobarbitalum as well as clobazam and biperideni lactas because of tremor, slowness of movement and impaired motor development. Moreover, arginine and cytriline supplementation as well as normal kaloric and protein diet were applied.

In addition to describing novel ALDH18A1 mutations our results extend the knowledge on the profile of pathogenicity of $P D K 3$ variants. $P D K 3$ encodes pyruvate dehydrogenase kinase isoform 3 and recently, based on a single family, the p.R158H missense mutation in this gene has been described as causing X-linked dominant Charcot-Marie-
Tooth disease-6. The prosed pathogenic mechanism was associated with increased enzyme activity conferred by the mutation (CMTX6; OMIM: 300905) (PubMed: 23297365). According to the ExAC database the PDK3 has relatively low tolerance of loss of function mutations (11 expected, one observed, $\mathrm{pLI}=0.88$ ) thus raising a possibility that loss of PDK3 function may also be pathogenic. We found the PDK3 p.Leu $71^{\star}$ mutation in our proband and his mother but not his affected brother. The p.Leu $71^{\star}$ mutation is predicted to cause nonsense mediated decay of mRNA thus leading to loss of function. The lack of any neurological symptoms in the mother indicates that loss of $P D K 3$ function is most likely not pathogenic in heterozygous females. Likewise, the lack of differences in diseases phenotype between the affected brothers discordant for the PDK3 p.Leu $71^{*}$ suggests that the mutation does not have a strong effect in hemizygous males, at least early in life.

In conclusion, our report broadens the spectrum of pathogenic recessive mutations causing $A L D H 18 A 1$ associated de Barsy syndrome. In addition, we provide evidence for non-pathogenicity of loss of function variants in $P D K 3$.

\section{Acknowledgement}

We thank the patients and their parents for their participation in this study.

\section{Conflicts of Interest}

There are no conflicts of interest.

Contributors RS: studied the patients, collected the samples, conceived the manuscript, supervised whole work, KS: studied the patients, helped in the clinical work, KK, TP: performed of the metabolic tests, AP, JK, GK: performed of the NGS study and bioinformatics processing of NGS raw data, MS: critically revised the manuscript, RP: supervised experimental work, analyzed the processed NGS data and critically revised the manuscript. Patient consent obtained. Competing interests none. Ethics approval Ethics approval was granted by the Institutional Review Board of Warsaw Medical University.

\section{References}

1. De Barsy AM, Moens E, Dierckx L (1967) Dwarfism, oligophrenia and elastic tissue hypoplasia: A new syndrome? Lancet 2: 47. 
Citation: Smigiel R, Kusmierska K, Pollak A, Szmyd K, Kosinska J, et al. (2017) Severe Phenotype of De Barsy Syndrome in Two Siblings with Novel Mutations in the ALDH18A1 Gene. J Clin Med Genomics 5: 145. doi: 10.4172/2472-128X.1000145

Page 4 of 4

2. Aldave AJ, Raber IM (2003) De Barsy Syndrome. In: NORD Guide to Rare Disorders. Lippincott Williams \& Wilkins. Philadelphia, PA, 6.

3. Kivuva EC, Parker MJ, Cohen MC, Wagner BE, Sobey G (2008) De Barsy syndrome: A review of the phenotype. Clin Dysmorphol 17: 99-107.

4. Guerra D, Fornieri C, Bacchelli B, Lugli L, Torelli P, et al. (2004) The De Barsy syndrome. J Cutan Pathol 31: 616-624.

5. Baumgartner MR, Rabier D, Nassogne MC, Dufier JL, Padovani JP, et al. (2005) Delta1-pyrroline-5-carboxylate synthase deficiency: Neurodegeneration, cataracts and connective tissue manifestations combined with hyperammonaemia and reduced ornithine, citrulline, arginine and proline. Eur J Pediatr 164: 31-36.

6. Bicknell LS, Pitt J, Aftimos S, Ramadas R, Maw MA, et al. (2008) A missense mutation in $A L D H 18 A 1$, encoding Delta1-pyrroline-5-carboxylate synthase (P5CS), causes an autosomal recessive neurocutaneous syndrome. Eur J Hum Genet 16: 1176-11864.

7. Skidmore DL, Chitayat D, Morgan T, Hinek A, Fischer B, et al. (2011) Further expansion of the phenotypic spectrum associated with mutations in ALDH18A1, encoding $\Delta^{1}$-pyrroline-5-carboxylate synthase (P5CS). Am J Med Genet A 155A: 1848-1856.

8. Zampatti S, Castori M, Fischer B, Ferrari P, Garavelli L, et al. (2012) De Barsy syndrome: A genetically heterogeneous autosomal recessive cutis laxa syndrome related to P5CS and PYCR1 dysfunction. Am J Med Genet A 158A: 927-931.
9. Morava E, Guillard M, Lefeber DJ, Wevers RA (2009) Autosomal recessive cutis laxa revisited. Eur J Hum Genet 17: 1099-1110.

10. McKenna A, Hanna M, Banks E, Sivachenko A, Cibulskis K, et al. (2010) The genome analysis toolkit: A MapReduce framework for analyzing nextgeneration DNA sequencing data. Genome Res 20: 1297-1303.

11. DePristo MA, Banks E, Poplin R, Garimella KV, Maguire JR, et al. (2011) A framework for variation discovery and genotyping using next-generation DNA sequencing data. Nat Genet 43: 491-498.

12. Wang K, Li M, Hakonarson $\mathrm{H}$, Annovar (2010) Functional annotation of genetic variants from high-throughput sequencing data. Nucleic Acids Res 38: e164.

13. Robinson JT, Thorvaldsdottir H, Winckler W, Guttman M, Lander ES, et al (2011) Integrative genomics viewer. Nat Biotechnol 29: 24-26.

14. Richards S, Aziz N, Bale S, Bick D, Das S, et al. (2015) Standards and guidelines for the interpretation of sequence variants: $A$ joint consensus recommendation of the American College of Medical Genetics and Genomics and the Association for Molecular Pathology. Genetics in Medicine 17: 405-423.

15. Fischer B, Callewaert B, Schröter P, Coucke PJ, Schlack C, et al. (2014) Severe congenital cutis laxa with cardiovascular manifestations due to homozygous deletions in ALDH18A1. Mol Genet Metab 112: 310-316.

16. Fischer-Zirnsak B, Escande-Beillard N, Ganesh J, Tan YX, Al Bughaili M, et al. (2015) Recurrent de novo mutations affecting residue Arg138 of pyrroline5 -carboxylate synthase cause a progeroid form of autosomal-dominant cutis laxa. Am J Hum Genet 97: 483-492. 